\title{
1 ISOT_Calc: a versatile tool for parameter estimation in sorption
}

\section{2 isotherms}

3 JOSÉ L. BELTRÁN ${ }^{\mathrm{a}}$, JOSEPH J. PIGNATELLO ${ }^{\mathrm{b}}$, MARC TEIXIDÓ $^{\mathrm{a}^{*}}$

4 aa Departament de Química Analítica, Universitat de Barcelona. Martí i Franqués, 1-11, 08028, 5 Barcelona, Spain.

$6 \quad{ }^{b}$ Department of Environmental Sciences, Connecticut Agricultural Experiment Station, 123

7 Huntington St., P.O. Box 1106, New Haven, Connecticut, 06504-1106, U.S.A.

8 *Corresponding author phone: +1-510-643-0733; Present address: Department of Civil and

9 Environmental Engineering, 410 O'Brien Hall, University of California at Berkeley, Berkeley, 10 CA 94720, U.S.A.

11 E-mail addresses: jlbeltran@ub.edu (J.L. Beltrán), joseph.pignatello@ct.gov (J.J. Pignatello), 12 mteixido@berkeley.edu (M. Teixidó).

\section{Abstract}

14 Geochemists and soil chemists commonly use parametrized sorption data to assess transport and 15 impact of pollutants in the environment. However, this evaluation is often hampered by a lack 16 of detailed sorption data analysis, which implies further non-accurate transport modeling. To 17 this end, we present a novel software tool to precisely analyze and interpret sorption isotherm data. Our developed tool, coded in Visual Basic for Applications (VBA), operates embedded

19 within the Microsoft Excel $^{\mathrm{TM}}$ environment. It consists of a user-defined function named 20 ISOT_Calc, followed by a supplementary optimization Excel macro (Ref_GN_LM). The 21 ISOT_Calc function estimates the solute equilibrium concentration in the aqueous and solid phases $\left(C_{e}\right.$ and $q$, respectively). Hence, it represents a very flexible way in the optimization of the sorption isotherm parameters, as it can be carried out over the residuals of $q, C_{e}$, or both simultaneously (i.e. orthogonal distance regression). The developed function includes the most 
usual sorption isotherm models, as predefined equations, as well as the possibility to easily

26 introduce custom-defined ones. Regarding the Ref_GN_LM macro, it allows the parameter

27 optimization by using a Levenberg-Marquardt modified Gauss-Newton iterative procedure. In

28 order to evaluate the performance of the presented tool, both function and optimization macro

29 have been applied to different sorption data examples described in the literature. Results showed

30 that the optimization of the isotherm parameters was successfully achieved in all cases,

31 indicating the robustness and reliability of the developed tool. Thus, the presented software tool,

32 available to researchers and students for free, has proven to be a user-friendly and an interesting

33 alternative to conventional fitting tools used in sorption data analysis.

\section{Keywords}

35 Sorption; Isotherm parameters; Nonlinear Least-squares; Excel; Orthogonal Distance

36 Regression; Levenberg-Marquardt 
51 Fate, reactivity and bioavailability of pollutants in the environment are mainly governed by 52 sorption phenomena, which exemplify the transfer of a solute from a mobile phase (liquid or 53 gaseous) to a sorbent solid phase. Thus, a detailed characterization of the sorption process is 54 essential to evaluate pollutant transport and potential risk for the environment (Boxall et al., 55 2003; Kümmerer, 2004). Sorption behavior at different solute concentration levels is commonly assessed by constructing a sorption isotherm at equilibrium conditions. So the solute sorbed concentration $(q)$ is represented as a function of the remaining aqueous concentration $\left(C_{e}\right)$.

58 Accordingly, the slope of each point in a sorption isotherm stands for the solid-liquid 59 distribution coefficient $\left(K_{\mathrm{d}}\right.$, Eq. 1), which provides the solid retention magnitude at a given 60 solute concentration $\left(q\right.$ or $\left.C_{e}\right)$ :

$61 \quad K_{d}=\frac{q}{C_{e}}$

62 Thus, the choice of an appropriate sorption isotherm is critical for accurate contaminant transport predictions (Hinz, 2001). Nevertheless, the sorption parametrization in most cases is

64 often imprecise because of the complex nature of the sorbent (i.e., soil), along with a misuse of 65 simple equations to attempt the data description. If the isotherm equation choice is 66 inappropriate, inaccurate results will lead to underestimate or overestimate the potential adverse 67 environmental effects. Eventually, this will negatively influence on the risk assessment studies needed for developing and implementing future regulations.

69 There are different equations which describe the sorption isotherms (Giles et al., 1974; 70 Goldberg, 2005; Hinz, 2001; Kinniburgh, 1986; Limousin et al., 2007), being the Langmuir and 71 Freundlich models the most used in the environmental sciences:

$72 q=S_{T} \cdot \frac{K_{L} \cdot C_{e}}{1+K_{L} \cdot C_{e}}$ 
74 In the Langmuir equation (Eq. 2), $S_{T}$ indicates the maximum sorption capacity, and $K_{L}$ is the

75 Langmuir affinity parameter. On the other hand, $K_{F}$ and $N$ stand for the Freundlich affinity

76 parameter and the exponential unitless term (Eq. 3), respectively. The determination of these

77 parameters from the experimental data pairs $C_{e} / q$ (usually obtained by batch equilibrium

78 methods) is straightforward in the cited models, as the Eq. 2 and 3 can be transformed to give

79 linear functions that can be analyzed by ordinary least-squares (OLS), as:

$80 \quad \frac{1}{q}=\frac{1}{S_{T}} \cdot \frac{1+K_{L} \cdot C_{e}}{K_{L} \cdot C_{e}}=\frac{1}{S_{T}}+\frac{1}{S_{T} \cdot K_{L}} \cdot \frac{1}{C_{e}}$

$81 \log (q)=\log \left(K_{F}\right)+N \cdot \log \left(C_{e}\right)$

82 However, when three or more parameter isotherm equations are needed to describe data, the 83 fitting of the model to experimental data requires the use of iterative non-linear least-squares 84 methods (NLLS)(Ho et al., 2002). Furthermore, it is widely accepted that linearization of 85 isotherm equations, when applicable, may lead to misleading results (Ho, 2004; Ho et al., 2005; 86 Motulsky and Christopoulos, 2004). To date, there are several alternatives to conduct this data 87 treatment. The possibilities range from specific programs, such as ISOFIT (Matott and Rabideau, 2008), to popular spreadsheets as MS-Excel -using the Solver add-in (Bolster and 89 Hornberger, 2007)- or multipurpose statistical/mathematical packages; e.g., Origin (OriginLab 90 Corp., Northampton, MA, USA), KaleidaGraph (Synergy Software, Reading, PA, USA), 91 Matlab (Mathworks, Natick, MA, USA) or SigmaPlot (Systat Software Inc., San Jose, CA, 92 USA) (Li et al., 2009; Sathishkumar et al., 2007; Xia and Pignatello, 2001).

93 In general, NLLS methods are based on the minimization of an objective function (U), which 94 depends on a set of parameters to be optimized. $U$ is defined as the sum of squared residual 95 errors $\left(e_{i}\right)$, obtained from the differences between an experimental magnitude (e.g., $\left.q_{\text {exp }, i}\right)$, and 
96 the corresponding values calculated on the basis of a guessed model (e.g., $q_{c a l c, i}$ ). For better

97 results, the use of statistical weights $\left(w_{i}\right)$ is recommended in the literature ${ }^{4}$ :

$98 \quad U=\sum_{i=1}^{n} w_{i} \cdot\left(e_{i}\right)^{2}=\sum_{i=1}^{n} w_{i} \cdot\left(q_{\mathrm{exp}, i}-q_{\text {calc }, i}\right)^{2}$

99 Where $q_{\text {exp }, i}$ and $q_{c a l c, i}$ indicate the experimental and calculated sorbed concentrations for each $i$ 100 data point, being $n$ the number of data points. The $q_{c a l c, i}$ values are determined from the 101 experimental (measured) equilibrium solute concentration in the liquid phase, the guessed 102 isotherm type and the corresponding parameters. For instance, in the Freundlich isotherm (Eq. 103 3), $q_{c a l c, i}$ takes the following form:

$q_{\text {calc }, i}=K_{F} \cdot C_{\exp , i}^{N}$

In an iterative NLLS procedure, the system is solved when a minimum in the objective function is attained $\left(\mathrm{U}_{\min }\right)$, providing the parameters that correspond to this $\mathrm{U}_{\min }$. To decide which type of isotherm best describes a given system, the user can try different isotherm equations, and apply different statistical tests over the results obtained. For example, the isotherm giving the lower

$109 \mathrm{U}_{\min }$ could be considered as the best, although other factors should be taken into account, as the 110 number of parameters to be optimized and their corresponding estimated standard deviations.

111 A different approach is proposed in this work, as we have developed a new function 112 (ISOT_Calc) which calculates the theoretical solute equilibrium concentrations in the solid and 113 liquid phases $\left(q_{T}\right.$ and $\left.C_{e, T}\right)$, for a given isotherm type (together with the corresponding 114 parameters) and the experimental conditions (mass of sorbent, liquid phase volume and initial 115 solute concentration). This is achieved by solving the mass-balance equation for each equilibrium point. Hence, the objective function to be minimized can be defined on the basis of

117 residuals in $C_{e}, q$, or both simultaneously. The function has been developed as a user-defined 118 function in the MS-Excel spreadsheet, and contains the most common isotherm models as 119 predefined equations. Moreover, "custom isotherms" can also be introduced by the user. The 120 optimization of the isotherm parameters can be carried out by using the Solver tool included in 
121 Excel, or by an Excel macro, also developed in this work (Ref_GN_LM), which is based on the

122 iterative Gauss-Newton Levenberg-Marquardt optimization algorithm.

123 The described procedure has been successfully tested with several sorption data described in the

124 literature. Specially, in the Excel versions corresponding to MS-Office 2007, 2010 and 2013 for

125 MS-Windows, as well as MS-Office 2011 for Apple Mac OSX (Further details about the

126 software, including requirements and availability can be found in Supplementary material of the

127 Appendix A). The results have been compared with those given in the literature, as well with

128 those obtained by the non-commercial program ISOFIT (Matott and Rabideau, 2008), and by

129 the graphing/data analysis commercial program KaleidaGraph (Synergy Software).

\section{$131 \quad 2$. Tool structure and mathematical background}

\section{2.1. Mass-balance solution}

133 The main core of the developed implementation is an Excel user-defined function named

134 "ISOT_Calc", written in MS Visual Basic for Applications (VBA), as the optimization routine

135 described below. It solves the mass-balance equations by a standard iterative Newton algorithm

136 (Demidovich and Maron, 1981), which calculates the theoretical solute concentration in both

137 solid and liquid phases ( $q_{T}$ and $C_{e, T}$, respectively) from a given isotherm equation, guessed

138 parameters and experimental conditions.

139 For illustration purposes, we will consider the case of a system which describes a Freundlich-

140 type isotherm. The initial experimental conditions in this system are: a volume of liquid phase

$141(V)$, an initial solute concentration $\left(C_{0}\right)$, and a sorbent mass $\left(M_{s}\right)$. After equilibration, the

142 remaining measured concentration in the liquid phase is $C_{e, e x p}$. The sorbed concentration is

143 calculated as:

$144 \quad q_{\exp }=V \frac{C_{0}-C_{e, \text { exp }}}{M_{s}}$ 
145 On the other hand, the calculated mass-balance using the Freundlich model (Eq. 3) can be 146 written as:

$$
V \cdot C_{0}=V \cdot C_{e, T}+M_{S} \cdot q_{T}=V \cdot C_{e, T}+M_{S} \cdot K_{F} \cdot C_{e, T}^{N}
$$

148 Where $V, C_{0}$ and $M_{s}$ are the independent variables, with known values; $K_{F}$ and $N$ are the guessed 149 parameter set of the model, and $C_{e, T}$ is the value to be calculated. This value can be determined 150 by the iterative Newton method, defining a function which is equal to zero when the system is 151 solved. This function, named $F$, is based on the difference between the experimental and 152 calculated mass-balances:

$$
F=V \cdot C_{0}-\left(V \cdot C_{e, T}+M_{S} \cdot K_{F} \cdot C_{e, T}^{N}\right)
$$

154 This equation is solved by successive approximations of $C_{e, T}$, being the value in the $n+1$ 155 iteration calculated from the previous one and the corresponding values of the $F$ function and its 156 derivative with respect to $C_{e, T}$. However, in order to avoid negative concentration values in the iterative process, the derivatives are taken respect to $\ln \left(C_{e, T}\right)$. In this way, the value computed in the $n+1$ iteration is obtained as:

$159 \ln \left(C_{e, T, n+1}\right)=\ln \left(C_{e, T, n}\right)-\frac{F_{(n)}}{F_{\ln (n)}^{\prime}}$

160 Where $F^{\prime}{ }_{l n}$ indicates the derivative of the $F$ function with respect to $\ln \left(C_{e, T}\right)$. The exponential 161 form of Eq. 11 gives the new value for $C_{e, T}$, which is always positive:

$162 C_{e, T, n+1}=\exp \left\{\ln \left(C_{e, T, n+1}\right)\right\}=\frac{C_{e, T, n}}{\exp \left\{F_{(n)} / F_{\ln (n)}^{\prime}\right\}}$

163 As the developed function contains several isotherm types, the procedure uses numerical 164 differentiation in order to maintain a reasonable length code. The iterative process ends when 165 the difference between experimental and calculated mass-balances is lower than a tolerance 166 limit; which has been established as $\leq 0.001 \%$ relative difference. This takes about $4-10$ 
167 iterations for each data point. The initial value used to start the iterative process is the total 168 concentration of the solute. This feature allows simulating theoretical isotherms without the 169 knowledge of the experimental solution equilibrium concentration $\left(C_{e, \text { exp }}\right)$.

170 The developed function includes 11 predefined models corresponding to the most common 171 isotherms used in solid-liquid equilibrium studies (Czinkota et al., 2002; Goldberg, 2005; Hinz, 172 2001; Kinniburgh, 1986; Xia and Pignatello, 2001). Table A1, given in the Appendix A, shows 173 the predefined models together with the corresponding number of parameters. Moreover, we 174 have included the possibility to enter directly a user-defined isotherm (type 0 ) in a cell of the 175 spreadsheet. This is achieved by the inclusion of a parser runtime subroutine in the VBA code 176 (Volpi, 2003), by substitution of the literal cell text into the corresponding numerical values. 177 Regarding isotherm parameters, they must be written as "P(1), $\mathrm{P}(2), \ldots$ ", and the solute concentration in liquid phase as "Ce". For example, a Freundlich isotherm introduced as user179 defined would take the form: "P(1)*Ce $\mathrm{e}^{\wedge} \mathrm{P}(2)$ " (without the quotation marks).

The algorithm described returns the expected $C_{e, T}$ as the primary result, along with $q_{T}$ as an intermediate calculation (see Eq. 9). In the Excel spreadsheet, both results can be easily retrieved by using the 'Index' function. For example, a call to the function as "=INDEX(ISOT_Calc(B16:D16;\$C\$8:\$C\$9;11;\$C\$6),1)" will return $C_{e, T}$. Nevertheless, if the last number (the index) is changed from 1 to 2 , the function will return $q_{T}$. The other terms in the user function are:

- B16:D16; standing for cells containing the $C_{0}, V, M_{\mathrm{s}}$, respectively.

- $\$ C \$ 8: \$ C \$ 9$; representing the parameter set of the guessed isotherm model. model (Eq. 3).

- \$C \$6; corresponding to a "dummy" cell, used only in custom isotherms (type 0) 
191 As ISOT_Calc requires a considerable amount of data input, a dialog box (developed using the 192 built-in VBA InputBox function) has been added to assists users (see Fig. 1).

\subsection{Optimization procedure}

One of the functions developed in this work consists of an optimization routine written as a macro (named ref_GN_LM), based on the Levenberg-Marquardt modification of the GaussNewton iterative algorithm (Johnson and Faunt, 1992). In each iteration, the isotherm parameters are shifted according with the expression:

$$
\left\{J^{T} J+\lambda \operatorname{diag}\left(J^{T} J\right)\right\} \Delta P=J^{T} \operatorname{Err}
$$

Where $J$ stands for the Jacobian matrix that contains, calculated by numerical differentiation, the partial derivatives of the errors with respect to the parameters. $\Delta P$ is the vector containing the shifts of parameters, $\lambda$ is the Marquardt parameter and Err indicates a vector containing the residual errors. The starting value for $\lambda$ is set to 10 , and it is decreased 10 -fold each successful iteration when the optimization process converges; if not, $\lambda$ is set equal to the number of consecutive non-convergence iterations. This procedure stops when the relative difference in the objective function (e.g., Eq. 6) between two consecutive iterative cycles is less than $0.1 \%$ and the Marquardt parameter is lower than 0.01 . In any case, the maximum number of iterations is limited to 20. To maintain similar magnitude orders of parameters during the refinement procedure, they are changed internally to their corresponding natural logarithms. In addition, this also avoids the possibility to obtain negative values. The routine allows estimating the errors in the parameters from the residuals and the shifting matrix (Johnson, 2000).

Although this corresponds to a standard optimization procedure, this work also provides a unique definition of the residuals regarding other procedures described in the introduction section. As noted previously, the mass balance procedure calculates the data pairs $\left(C_{e, T}\right.$ and $\left.q_{T}\right)$ for each equilibrium point from the experimental conditions and guessed isotherm parameters. 
216 This allows defining the residual errors in $C_{e}, q$, or distance (d) between the calculated and 217 experimental points:

$$
\Delta \mathrm{C}_{\mathrm{e}, \mathrm{i}}=\mathrm{C}_{\mathrm{e}, \exp , \mathrm{i}}-\mathrm{C}_{\mathrm{e}, \mathrm{T}, \mathrm{i}} \quad \Delta \mathrm{q}_{\mathrm{i}}=\mathrm{q}_{\text {exp } \mathrm{i}}-\mathrm{q}_{\mathrm{T}, \mathrm{i}}
$$

$$
\left.\mathrm{d}_{\mathrm{i}}=\sqrt{\left(\mathrm{C}_{\mathrm{e}, \mathrm{exp}, \mathrm{i}}-\mathrm{C}_{\mathrm{e}, \mathrm{T}, \mathrm{i}}\right)^{2}+\left(\mathrm{q}_{\mathrm{exp}, \mathrm{i}}-\mathrm{q}_{\mathrm{T}, \mathrm{i}}\right)^{2}}=\sqrt{\left(\Delta \mathrm{C}_{\mathrm{i}}\right)^{2}+\left(\Delta \mathrm{q}_{\mathrm{i}}\right)^{2}}\right\}
$$

220 Differences in the definition of the errors are shown in Fig. 2. This figure represents a 221 hypothetical isotherm with its experimental data points (symbolized as diamonds), together with 222 a solid line which stands for the ISOT_Calc function calculated points from the Freundlich equation (Eq. 3). One data point is detailed in the zoomed in section, indicating the errors in $q$ and $C_{e}$ (Eq. 14) and the $d$ distance between an experimental and a circle-shaped calculated point (Eq. 15). Additionally, Fig. 2 also shows the error $\left(\Delta \mathrm{q}_{1}\right)$ obtained by a conventional procedure (e.g., using the Freundlich model in Eq. 6).

Although the errors are calculated as described, the objective functions to be minimized in the optimization procedure use weighting factors to allow all data points to be equally significant. This is particularly interesting when dealing with pollutant sorption onto natural sorbents. As most environmentally relevant concentrations fall within the trace levels, accurate modeling in the lower region of the isotherm is crucial. Thus, the objective functions for minimization of the errors in $C_{e}$ and $q$ are calculated from the corresponding relative errors. This is equivalent to use weighting factors equal to the inverse of the squared measured values (e.g., $w_{i}=1 /\left(C_{e, e x p, i}\right)^{2}$ or $\left.w_{i}=1 /\left(q_{\text {exp }, i}\right)^{2}\right):$

$U_{C e}=\sum_{i=1}^{n} \frac{1}{C_{e, \exp , i}^{2}} \Delta C_{e, i}^{2}=\sum_{i=1}^{n}\left(\frac{\Delta C_{e, i}}{C_{e, \exp , i}}\right)^{2}$

$$
U_{q}=\sum_{i=1}^{n} \frac{1}{q_{\mathrm{exp}, i}^{2}} \Delta q_{i}^{2}=\sum_{i=1}^{n}\left(\frac{\Delta q_{i}}{q_{\mathrm{exp}, i}}\right)^{2}
$$


237 On the other hand, the definition of residual errors as the distance between experimental and 238 theoretical points allows an easy implementation of the orthogonal distance regression. This 239 possibility, pointed by Poch and Villaescusa (2012), has been satisfactorily applied to 240 theoretical Langmuir isotherms for determining the isotherm parameters. In fact, in a "classical" 241 procedure for modeling sorption data, it is assumed that $C_{e}$ is the independent variable (error242 free), and the isotherm parameters are calculated in order to minimize the errors in the 243 dependent variable $(q)$. The orthogonal distance regression takes into account the errors in both 244 variables $\left(C_{e}\right.$ and $q$ ), and the weighted objective function $\left(U_{d}\right)$ to be minimized is defined as a 245 combination of Eq. 16 and 17:

$246 \quad U_{d}=\sum_{i=1}^{n}\left\{\left(\frac{\Delta C_{e, i}}{C_{e, \mathrm{exp}, i}}\right)^{2}+\left(\frac{\Delta q_{i}}{q_{\mathrm{exp}, i}}\right)^{2}\right\}$

247 As the $C_{e, T, i}$ and $q_{T, i}$ values are directly obtained using the ISOT_Calc function, the calculation 248 of the different objective functions (Eq. 16 to 18) is straightforward. Table A2 shows a possible 249 arrangement of a worksheet, indicating the experimental data, the calculated $C_{e, T, i}$ and $q_{T, i}$ values 250 and the individual errors.

251 The optimization of the isotherm parameters is an easy task using the macro ref_GN_LM. Once 252 started, it requires the residual errors $\left(\Delta C_{e} / C_{e}\right.$, for example), the parameters to be optimized, 253 and a starting cell to print the results. The results include the final values of the parameters 254 together with their estimated standard deviations -the standard deviation (SD) and relative 255 standard deviation (RSD), respectively-. Moreover, they will also contain other statistical 256 parameters, such as the sum of weighted squared residuals (Eq. 16, 17 or 18), the mean 257 weighted square error (MWSE) and the root mean square error (RMSE):

$M W S E=U /(n-p) \quad R M S E=\sqrt{M W S E}$

259 Where $p$ indicates the number of parameters refined and $\mathrm{n}$ stands for the number of 260 experimental points. All these statistical values taken together, along with the estimated errors 
in the isotherm parameters, are very useful to decide which isotherm equation fits better to a set

262 of experimental data points.

263 The use of the Solver function in the Excel spreadsheet allows another possible optimization of

264 the isotherm parameters. In this case, the user defines a cell containing the value of the objective

265 function (value to be minimized, corresponding to the sum of squared errors) and the parameters

266 to optimize. Although the results obtained are similar to those obtained with ref_GN_LM

267 macro, this latter is significantly faster. Moreover, as Solver function does not provide the

268 estimated errors of the optimized parameters, they should be calculated using another routine;

269 e.g., Solvstat for Excel (Billo, 2001).

\section{Results and discussion}

To test the calculation procedures, we have applied the described routines to determine the isotherm parameters in several sorption examples found in the literature. In order to be able to compare results, we have calculated the corresponding parameters by using the different optimization possibilities included in the ISOT_Calc/Ref_GN_LM procedure (i.e., minimizing $U_{C e}, U_{q}$ and $U_{d}$; as described in Eqs. 16-18), and also by using the ISOFIT (Matott and Rabideau, 2008) and KaleidaGraph (Synergy Software) programs. Both ISOFIT and KaleidaGraph minimize the squared differences between $\mathrm{q}_{\exp }$ and $\mathrm{q}_{\mathrm{cal}}$, this latter calculated after the proposed isotherm equation and the experimental $C_{e}$ values. To get comparable results, we have used similar weighting factors $\left(\mathrm{w}_{\mathrm{i}}=1 /\left(\mathrm{q}_{\mathrm{exp}, \mathrm{i}}\right)^{2}\right)$ in both programs.

The first tested system (System I) corresponds to the sorption of tetracycline onto a natural loamy soil (Teixidó et al., 2012). The 16 experimental data points are shown on the left side of Fig. 3. The shape of the isotherm suggests a Langmuir-type isotherm, as indicated in the reference. Results are given in Table 1 (as System I). They include the parameters for the tested isotherm equations in each calculation mode, together with their estimated errors (SD values) and MWSE values. In each case, the MWSE for the Freundlich model is 2-3 times higher than 
those corresponding to the Langmuir model, clearly confirming that this latter is a better model for describing the data. It should also be noted that the MWSE values depend on the calculation procedure. For instance, ISOT_Calc uses a different residual definition (as indicated in Eq. 14 and 15) than KaleidaGraph and ISOFIT. In agreement with the obtained error values, the good fit of the Langmuir model to experimental data is also shown on left side of Fig. 3, where both experimental and calculated isotherms are plotted. In addition, the right side of this figure also depicts an acceptable distribution of the errors $\left(\Delta C_{e} / C e\right.$ and $\Delta q / q$, as squares and triangles, respectively) obtained when the objective function is $U_{d}$. As it can be noticed by the reader, this figure allows an easy identification of the outliers or the worst fitted experimental points.

The second system (System II) deals with a 10-experimental point data set that has been taken from Kinniburgh (1986). It describes the sorption of $\mathrm{Zn}(\mathrm{II})$ ions onto ferrihydrite (an iron hydrous oxide gel). Although the sorption data were firstly attributed to a Langmuir-type isotherm by the authors, Matott and Rabideau (2008) showed that the Freundlich equation gave better fitting results (9-times lower sum of squared residuals). Other tested isotherm models with three parameters (Toth, Polanyi, etc.) were also evaluated in this work. They lowered the objective function, but in a lesser extension. Thus, we have compared the Langmuir and Freundlich models on this data set. In all calculations (using ISOFIT, KaleidaGraph and the different ISOT_Calc/Ref_GN_LM modes), the MWSE values obtained for the Freundlich model were about 7-9 times lower than in the Langmuir model (see "System II", in Table 1).

Regarding the third studied system (System III), this describes the sorption of trichloromethane onto a soil with a high content of natural organic matter (Xia and Pignatello, 2001). The authors tested several isotherm models (Freundlich, Dual Mode -DMM- and Polanyi-Manes) to fit the 107 experimental data points. Although the Polanyi-Manes model gave a lower MWSE value than the obtained with the DMM (0.0051 and 0.0070, respectively), it was considered not appropriate to explain the experimental data. Otherwise, it can also be argued that the small difference in MWSE is attributed to a higher number of adjustable parameters (4 and 3 used in 313 the Polanyi-Manes model and DMM, respectively). Besides, although more adjustable 
314 parameters sometimes may achieve better data fittings, it is recommended use simpler isotherm 315 equations (i.e., with lesser number of parameter equations). Thus, we have compared the results 316 obtained with the Freundlich and Dual Mode models. In the original article, the isotherm 317 parameters were determined either using the linearized form of the Freundlich model (as in Eq.

318 5) or NLLS regression in the DMM case. In this latter case, the objective function was 319 equivalent to the indicated in Eq. 6, being $w_{i}=\left(1 / q_{\text {exp }}\right)^{2}$. Results for system III are also presented in Table 1. Although different calculation procedures have been used, the calculated isotherm parameters are very similar, both for the Freundlich model and DMM (i.e., differences lie near the estimated errors of the parameters). In all cases, the MWSE values for DMM were about the half of the corresponding obtained for the Freundlich model, in agreement with the results given by Xia and Pignatello (2001).

As exemplified by Table 1, good fittings have been successfully achieved in the three examples. Nevertheless, we have also conducted a test to evaluate the ability of the optimization procedure in system III. Although the corresponding parameters for the DMM isotherm are already known $-\mathrm{K}_{\mathrm{D}}=10.6, \mathrm{~S}^{0}=13.3$ and $\mathrm{b}=1.3-$ we have introduced deliberately bad starting values; such as $1000,1,0.001$ and combinations of them. Table 2 lists the parameter sets used as initial values and the optimized values obtained using the different objective functions. It also includes the number of iterations needed to achieve the final values. Results show that despite the wide range of initial estimates, the final values are very similar (for each objective function), being the differences within their estimated SD. This visibly indicates the robustness against bad estimates of the optimization procedure.

\section{Conclusions}

The combination of the ISOT_Calc/Ref_GN_LM (developed as function and macro for MS-

337 Excel, respectively) has proven to be a useful tool in the evaluation of the isotherm parameters 338 from sorption data. The function ISOT_Calc enables MS-Excel to calculate the theoretical 339 equilibrium concentrations in the liquid and solid phases $\left(C_{e, T}\right.$ and $\left.q_{T}\right)$ for a guessed isotherm 
equation (together with its parameters), at the given experimental conditions ( $V, M_{\mathrm{S}}$ and $C_{0}$ ).

341 This feature can be used for two different purposes; the main is for the optimization of the 342 isotherm parameters (fitting the model to experimental data), but it can also be used for isotherm simulation. The developed function includes as predefined models the most common used isotherms. Moreover, user-defined isotherms can also be easily applied.

Regarding the developed macro Ref_GN_LM, it allows refining the isotherm parameters. This optimization can be carried out over the residuals defined in either $C_{e}$ or $q$, or a combination of both as well. This allows minimizing the orthogonal distance between the calculated and minimization is carried out only over the sorbed concentration $(q)$. The implementation of this macro, based on the Levenberg-Marquardt procedure, has proven to be robust and reliable. Although the optimization could also be carried out using the MS-Excel Solver tool, the routine described here is faster. On the other hand, Ref_GN_LM is an independent macro, allowing the refinement of the parameters of other functions, as a stand-alone NLLS regression method.

One of the main features of the ISOT_Calc/Ref_GN_LM procedure is its integration in the Excel spreadsheet. This means that sorption data analysis and graphical interpretation (e.g., editing or plotting of experimental data and calculated results) are easy tasks, even for low-level latter plot allows testing if residuals are randomly distributed or if systematic errors exist.

All the above mentioned capabilities make the ISOT_Calc/Ref_GN_LM a versatile tool in the study of sorption isotherms. The comparison between different models is straightforward, as it can be attained with statistical parameters (MWSE or RMSE) and visual inspection of 
experimental/calculated isotherms or residual distribution plots. All these options taken together

367 have proven to be remarkably interesting in assessing sorption in complex scenarios (e.g.,

368 natural soils). Finally, the results obtained when applying our developed procedure to literature

369 data are virtually the same as published, and those obtained with well-established programs

370 such as ISOFIT and KaleidaGraph.

\section{ACKNOWLEDGEMENTS}

372 This research was funded by the Spanish Ministerio de Ciencia y Tecnología (Project 373 CTQ2010-20156), the U.S. Department of Agriculture, NIFA, and the National Science

374 Foundation CBET 0853682. Authors especially thank Frank Ferrandino for his valuable 375 comments about this work. Authors also thank Jordi Montornés and Sergi Marí for their kind 376 contributions to the tutorial video. Marc Teixidó thanks Universitat de Barcelona for the APIF 377 grant.

\section{APPENDIX A. SUPPLEMENTARY MATERIAL}

379 Further details associated with the present work can be found in the appendix A file. This 380 Supplementary material includes: a software description (including requirements and 381 availability); a list of the predefined isotherms in the ISOT_Calc function; and a suggested 382 example of isotherm data and calculations.

APPENDIX B. SUPPLEMENTARY MATERIAL

Appendix B includes an easy-to-follow tutorial video (length: 9 min, $35 \mathrm{sec}$ ) showing how to use the ISOT_Calc/Ref_GN_LM procedure. Specially, this video details how to fit experimental data using predefined isotherm equations and user-defined isotherm equations. This material is available free of charge via the Internet. 
Billo, E.J., 2001. Excel for Chemists: a Comprehensive Guide, 2nd edn. Wiley-VCH, New York, NY, $760 \mathrm{pp}$.

Bolster, C.H., Hornberger, G.M., 2007. On the use of linearized Langmuir equations. Soil Science Society of America Journal 71, 1796-1806. doi:10.2136/ssaj2006.0304

Boxall, A.B., Kolpin, D.W., Halling-Sorensen, B., Tolls, J., 2003. Are veterinary medicines causing environmental risks? Environmental Science \& Technology 37, 286A-294A. doi: $10.1021 / \mathrm{es} 032519 \mathrm{~b}$

Czinkota, I., Foldenyi, R., Lengyel, Z., Marton, A., 2002. Adsorption of propisochlor on soils and soil components equation for multi-step isotherms. Chemosphere 48, 725-731. doi:S0045-6535(02)00139-X

Demidovich, B.P., Maron, I.A., 1981. Computational Mathematics, 3rd print. edn. Mir, Moscow, $688 \mathrm{pp}$.

Giles, C.H., Smith, D., Huitson, A., 1974. General treatment and classification of solute adsorption-isotherm. 1. Theoretical . Journal of Colloid and Interface Science 47, 755-765. doi:10.1016/0021-9797(74)90252-5

Goldberg, S., 2005. Equations and models describing adsorption processes in soils, in: Tabatabai, M.A, Sparks, D.L. (Eds), Chemical processes in soils. Soil Science Society of America, Madison, pp. 489-517.

Hinz, C., 2001. Description of sorption data with isotherm equations. Geoderma 102, 405-406. doi:10.1016/S0016-7061(00)00071-9

Ho, Y.S., 2004. Selection of optimum sorption isotherm. Carbon 42, 2115-2116. doi:10.1016/j.carbon.2004.03.019

Ho, Y.S., Chiu, W.T., Wang, C.C., 2005. Regression analysis for the sorption isotherms of basic dyes on sugarcane dust. Bioresource Technology 96, 1285-1291. doi:10.1016/j.biortech.2004.10.021

Ho, Y.S., Porter, J.F., McKay, G., 2002. Equilibrium isotherm studies for the sorption of divalent metal ions onto peat: Copper, nickel and lead single component systems. Water, Air, \& Soil Pollution 141, 1-33. doi:10.1023/a:1021304828010

Johnson, M.L., 2000. Parameter correlations while curve fitting. Methods in Enzymology 321, 424-446. doi:S0076-6879(00)21207-X

Johnson, M.L., Faunt, L.M., 1992. Parameter estimation by least-squares methods. Methods in Enzymology 210, 1-37. doi:0076-6879(92)10003-V

Kinniburgh, D.G., 1986. General purpose adsorption isotherms. Environmental Science \& Technology 20, 895-904. DOI: 10.1021/es00151a008

Kümmerer, K., 2004. Pharmaceuticals in the Environment: Sources, Fate, Effects and Risks, 2nd edn. Springer-Verlag, New York, NY, 528 pp. 
Li, R., Wen, B., Zhang, S., Pei, Z., Shan, X., 2009. Influence of organic amendments on the sorption of pentachlorophenol on soils. Journal of Environmental Sciences 21, 474-480. doi:10.1016/S1001-0742(08)62294-9

Limousin, G., Gaudet, J.P., Charlet, L., Szenknect, S., Barthes, V., Krimissa, M., 2007. Sorption isotherms: A review on physical bases, modeling and measurement. Applied Geochemistry 22, 249-275. doi:10.1016/j.apgeochem.2006.09.010

Matott, L.S., Rabideau, A.J., 2008. ISOFIT - A program for fitting sorption isotherms to experimental data. Environmental Modelling \& Software 23, 670-676. doi:10.1016/j.envsoft.2007.08.005

Motulsky, H., Christopoulos, A., 2004. Fitting Models to Biological Data using Linear and Nonlinear Regression: a Practical Guide to Curve Fitting. Oxford University Press, New York, NY, $352 \mathrm{pp}$.

Poch, J., Villaescusa, I., 2012. Orthogonal Distance Regression: A Good Alternative to Least Squares for Modeling Sorption Data. Journal of Chemical and Engineering Data 57, 490499. doi:10.1021/je201070u

Sathishkumar, M., Binupriya, A.R., Vijayaraghavan, K., Yun, S.-I., 2007. Two and threeparameter isothermal modeling for liquid-phase sorption of Procion Blue H-B by inactive mycelial biomass of Panus fulvus. Journal of Chemical Technology and Biotechnology 82, 389-398. doi:10.1002/jctb.1682

\section{Synergy Software. http://www.synergy.com/wordpress_650164087/kaleidagraph/} (accessed Dec 2015).

Teixidó, M., Granados, M., Prat, M.D., Beltrán, J.L., 2012. Sorption of tetracyclines onto natural soils: data analysis and prediction. Environmental Science and Pollution Research 19, 3087-3095. doi:10.1007/s11356-012-0954-5

Volpi, L., 2003. Runtime Math Formulas Evaluation in Excel. http://digilander.libero.it/foxes/mathparser/Run_Time_\%20Math_Formula_\%20Excel.htm (accessed Dec 2015).

Xia, G., Pignatello, J.J., 2001. Detailed sorption isotherms of polar and apolar compounds in a high-organic soil. Environmental Science \& Technology 35, 84-94.

doi: $10.1021 / \mathrm{es} 0013201$ 
459 Fig. 1. Dialog box to enter experimental values and isotherm equation in ISOT_Calc.

460 Fig. 2. Definition of errors in experimental data.

461 Fig. 3. Left: Sorption isotherm of tetracycline onto a loamy soil. The solid line stands for the 462 Langmuir model fit. Data from Teixidó et al. (2012). Right: Distribution of residuals from the 463 relative errors in $C_{\mathrm{e}}$ (squares) and q (triangles). 


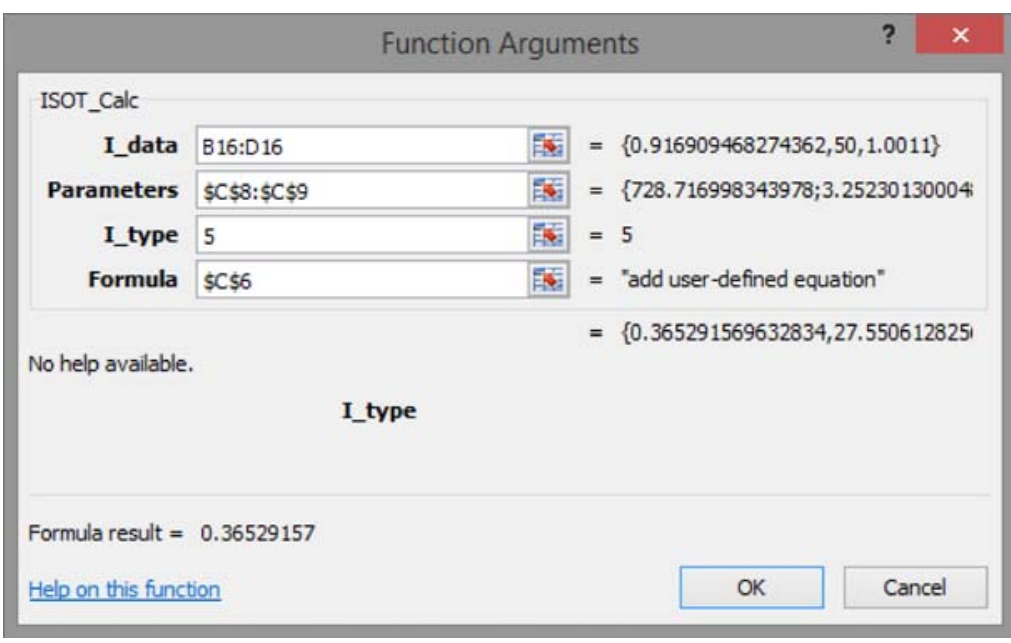

475 Fig. 1. Dialog box to enter experimental values and isotherm equation in ISOT_Calc.

476 


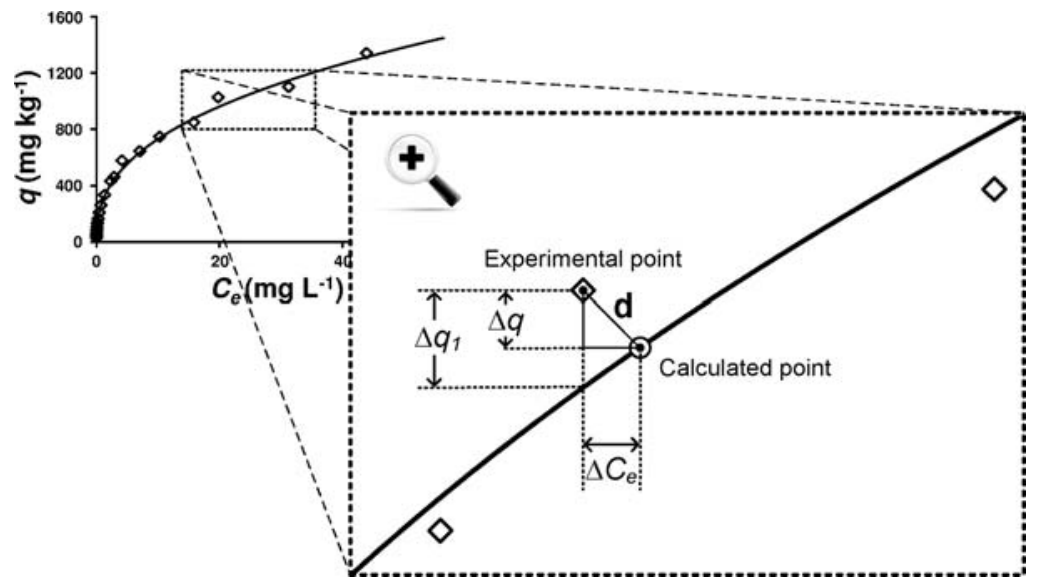

481 Fig. 2. Definition of errors in experimental data. 

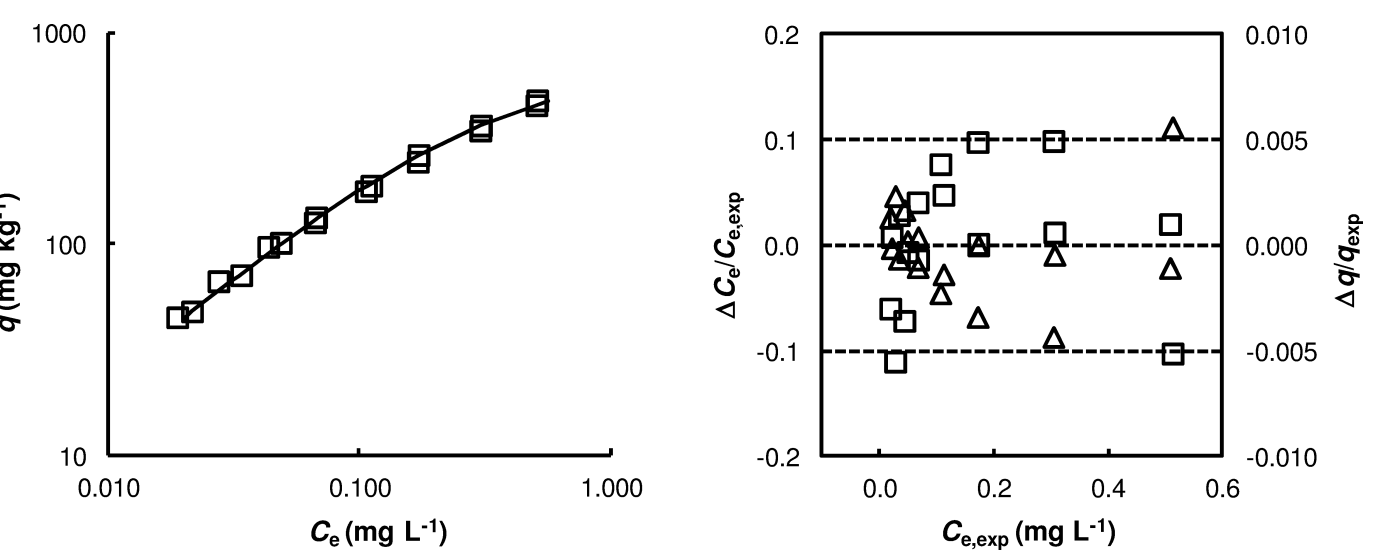

485 Fig. 3. Left: Sorption isotherm of tetracycline onto a loamy soil. The solid line stands for the 486 Langmuir model fit. Data from Teixidó et al. (2012). Right: Distribution of residuals from the 487 relative errors in $C_{\mathrm{e}}$ (squares) and q (triangles). 
Table 1. Isotherm parameters for Systems I, II and III. The estimated standard deviations are given between parentheses.

\begin{tabular}{|c|c|c|c|c|c|c|c|c|c|c|}
\hline \multirow[b]{2}{*}{ Parameter } & \multicolumn{3}{|c|}{ Langmuir model } & \multicolumn{3}{|c|}{ Freundlich model } & \multicolumn{4}{|c|}{ Dual Mode model (DMM) } \\
\hline & $S_{T}$ & $K_{L}$ & $\begin{array}{l}M W S E \\
\left(\mathrm{x} 10^{-4}\right)\end{array}$ & $K_{F}$ & $N$ & $\begin{array}{l}M W S E \\
\left(\times 10^{-4}\right) \\
\end{array}$ & $K_{D}$ & $S^{0}$ & $b$ & $\begin{array}{l}W W E \\
\left(\times 10^{-4}\right) \\
\end{array}$ \\
\hline \multicolumn{11}{|l|}{ System I } \\
\hline Lit. values $^{\mathrm{a}}$ & 693(27) & $3.5(0.3)$ & 23 & $828(36)$ & $0.71(0.03)$ & 61 & - & - & - & - \\
\hline ISOFIT $^{\mathrm{b}}$ & $690(31)$ & $3.5(0.2)$ & 22 & $817(39)$ & $0.71(0.02)$ & 65 & - & - & - & - \\
\hline KaleidaGraph ${ }^{\mathrm{b}}$ & $690(32)$ & $3.5(0.2)$ & 22 & $817(40)$ & $0.71(0.02)$ & 65 & - & - & - & - \\
\hline ISOT_Calc ${ }^{c}$ & $730(26)$ & $3.2(0.2)$ & 9 & $849(46)$ & $0.72(0.02)$ & 93 & - & - & - & - \\
\hline ISOT_Calc $^{\mathrm{d}}$ & $757(25)$ & $3.0(0.2)$ & 0.05 & $775(30)$ & $0.68(0.02)$ & 0.1 & - & - & - & - \\
\hline ISOT_Calc $^{\mathrm{e}}$ & $729(22)$ & $3.3(0.2)$ & 39 & $848(43)$ & $0.72(0.02)$ & 94 & - & - & - & - \\
\hline \multicolumn{11}{|l|}{ System II } \\
\hline Lit. values ${ }^{\mathrm{f}}$ & 7.98 & 3.10 & & & & & & & & \\
\hline ISOFIT $^{\mathrm{b}}$ & $8.1(0.7)$ & $3.0(0.4)$ & 40 & $8.5(0.1)$ & $0.682(0.009)$ & 6 & - & - & - & - \\
\hline KaleidaGraph $^{\mathrm{b}}$ & $8.1(0.7)$ & $3.0(0.4)$ & 40 & $8.5(0.1)$ & $0.682(0.009)$ & 6 & - & - & - & - \\
\hline ISOT_Calc ${ }^{c}$ & $8.0(0.7)$ & $3.1(0.4)$ & 18 & $8.5(0.2)$ & $0.687(0.009)$ & 2 & - & - & - & - \\
\hline ISOT $^{-} \mathrm{Calc}^{\mathrm{d}}$ & $8.8(0.6)$ & $2.6(0.3)$ & 6 & $8.4(0.1)$ & $0.677(0.010)$ & 1 & - & - & - & - \\
\hline ISOT_Calc $^{\mathrm{e}}$ & $8.2(0.6)$ & $2.9(0.3)$ & 25 & $8.5(0.2)$ & $0.684(0.009)$ & 3 & - & - & - & - \\
\hline \multicolumn{11}{|l|}{ System III } \\
\hline Lit. values ${ }^{\mathrm{g}}$ & - & - & - & $18.6(0.2)$ & $0.921(0.002)$ & 134 & $10.6(0.2)$ & $13.3(1.4)$ & $1.3(0.2)$ & 70 \\
\hline ISOFIT $^{\mathrm{b}}$ & - & - & - & $18.3(0.2)$ & $0.920(0.002)$ & 129 & $10.6(0.1)$ & $13.3(1.4)$ & $1.3(0.2)$ & 69 \\
\hline KaleidaGraph $^{\mathrm{b}}$ & - & - & - & $18.3(0.2)$ & $0.920(0.002)$ & 131 & $10.6(0.1)$ & $13.2(1.4)$ & $1.3(0.2)$ & 72 \\
\hline ISOT $\mathrm{Calc}^{\mathrm{c}}$ & - & - & - & $18.9(0.2)$ & $0.916(0.002)$ & 14 & $10.9(0.2)$ & $10.1(1.0)$ & $1.8(0.2)$ & 9 \\
\hline ISOT_Calc ${ }^{\mathrm{d}}$ & - & - & - & $18.1(0.3)$ & $0.924(0.003)$ & 62 & $10.6(0.1)$ & $14.8(1.6)$ & $1.2(0.2)$ & 31 \\
\hline ISOT_Calc ${ }^{\mathrm{e}}$ & - & - & - & $18.5(0.3)$ & $0.921(0.002)$ & 79 & $10.7(0.1)$ & $13.6(1.4)$ & $1.3(0.2)$ & 41 \\
\hline
\end{tabular}

${ }^{a}$ : from Teixidó et al. (2012)

${ }^{\mathrm{b}}$ : calculated using Eq. 6 , where $\mathrm{w}_{\mathrm{i}}=\left(1 /\left(\mathrm{q}_{\mathrm{exp}, \mathrm{i}}\right)\right)^{\wedge} 2$

${ }^{\text {c}}$ : calculated using Eq. 16, ${ }^{\text {d: }}$ calculated using Eq. 17 and $^{\text {e }}$ : calculated using Eq. 18

: from Kinninburg (1986)

g: from Xia and Pignatello (2001) 
Table 2. Comparison of the optimized isotherm parameters in system III using different initial values (DMM sorption isotherm).

\begin{tabular}{|c|c|c|c|c|c|c|c|c|c|c|c|c|c|c|}
\hline \multicolumn{3}{|c|}{ Starting values } & \multicolumn{12}{|c|}{ Final values after parameter optimization } \\
\hline \multirow[b]{2}{*}{$\mathrm{K}_{\mathrm{D}}$} & \multirow[b]{2}{*}{$\mathrm{S}^{0}$} & \multirow[b]{2}{*}{$\mathrm{b}$} & \multicolumn{4}{|c|}{ Objective function: $\mathrm{U}_{\mathrm{Ce}}$} & \multicolumn{4}{|c|}{ Objective function: $\mathrm{U}_{\mathrm{q}}$} & \multicolumn{4}{|c|}{ Objective function: $\mathrm{U}_{\mathrm{d}}$} \\
\hline & & & $\mathrm{K}_{\mathrm{D}}$ & $\mathrm{S}^{0}$ & $\mathrm{~b}$ & $\mathrm{Nit}^{\mathrm{a}}$ & $\mathrm{K}_{\mathrm{D}}$ & $S^{0}$ & $\mathrm{~b}$ & $\mathrm{Nit}^{\mathrm{a}}$ & $\mathrm{K}_{\mathrm{D}}$ & $\mathrm{S}^{0}$ & $\mathrm{~b}$ & $\mathrm{NIt}^{\mathrm{a}}$ \\
\hline 0.001 & 0.001 & 0.001 & 10.93 & 10.26 & 1.77 & 9 & 10.60 & 15.09 & 1.14 & 10 & 10.65 & 13.58 & 1.30 & 10 \\
\hline 1 & 1 & 1 & 10.94 & 10.12 & 1.80 & 7 & 10.60 & 14.80 & 1.16 & 7 & 10.65 & 13.31 & 1.33 & 7 \\
\hline 1000 & 1000 & 1000 & 10.93 & 10.27 & 1.77 & 9 & 10.60 & 15.10 & 1.13 & 8 & 10.65 & 13.58 & 1.30 & 9 \\
\hline 1000 & 1 & 1 & 10.94 & 10.12 & 1.80 & 10 & 10.60 & 14.86 & 1.16 & 11 & 10.65 & 13.31 & 1.33 & 10 \\
\hline 1 & 1000 & 1 & 10.93 & 10.28 & 1.77 & 9 & 10.60 & 15.09 & 1.14 & 9 & 10.64 & 13.66 & 1.29 & 9 \\
\hline 1 & 1 & 1000 & 10.94 & 10.13 & 1.80 & 8 & 10.60 & 14.86 & 1.16 & 8 & 10.65 & 13.42 & 1.32 & 9 \\
\hline 1 & 0.001 & 0.001 & 10.94 & 10.13 & 1.80 & 9 & 10.60 & 15.08 & 1.14 & 11 & 10.65 & 13.57 & 1.30 & 12 \\
\hline 0.001 & 1 & 0.001 & 10.93 & 10.26 & 1.77 & 10 & 10.60 & 15.15 & 1.13 & 9 & 10.64 & 13.64 & 1.29 & 10 \\
\hline 0.001 & 0.001 & 1 & 10.93 & 10.28 & 1.77 & 9 & 10.60 & 15.11 & 1.13 & 9 & 10.65 & 13.40 & 1.32 & 10 \\
\hline
\end{tabular}

${ }^{a}$ : number of iterations until convergence 
Experimental point

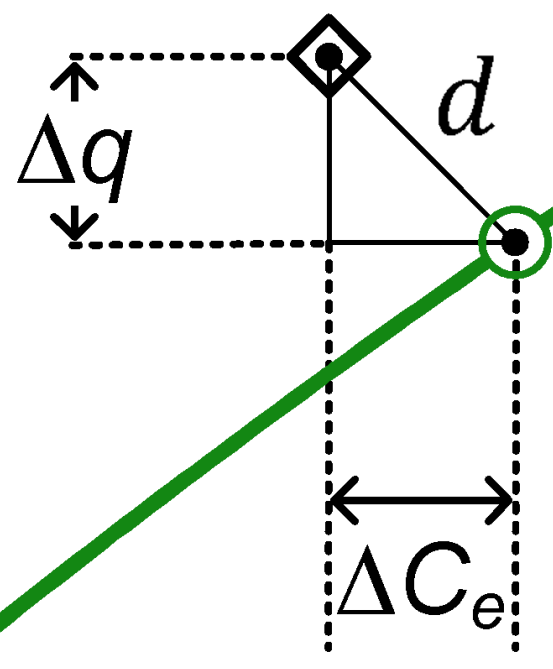

Calculated point

$$
d_{i}=\sqrt{\left(\Delta C_{e, i}\right)^{2}+\left(\Delta q_{i}\right)^{2}}
$$

